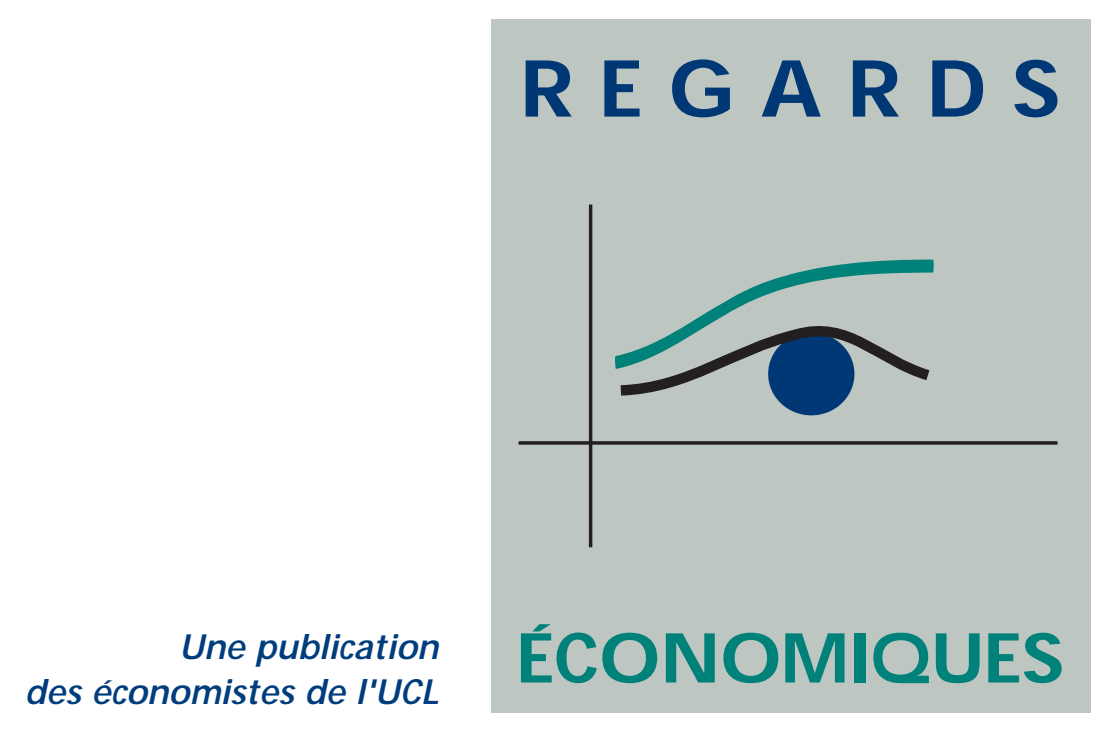

Juin 2008 - Numéro 60

\title{
La libéralisation du secteur postal
}

En 2011, le marché postal sera entièrement libéralisé. Le service universel sera-t-il maintenu? Comment sera-t-il financé ? Dans cet article, nous abordons la question du financement du service universel postal dans un environnement concurrentiel.

La Commission européenne propose plusieurs pistes, les Etats Membres doivent maintenant choisir la solution la plus appropriée à leur marché.

\section{La concurrence dans le secteur postal}

\section{Axel Gautier}

En Europe, la libéralisation du secteur postal a commencé en 1997. La première directive postale réaffirme l'importance des obligations de service universel (distribution et collecte journalière sur l'ensemble du territoire, prix abordable, points de contacts accessibles, ...) et, parallèlement à cela, prévoit l'ouverture graduelle du marché à la concurrence, dans un premier temps pour les envois de plus de 350gr. En 2002, la deuxième directive postale accélère le processus de libéralisation en réduisant progressivement la taille du secteur réservé où l'opérateur historique est protégé de la concurrence. Depuis le 1er janvier 2006, le secteur réservé est limité aux envois de moins de 50gr Finalement, la troisième directive postale, publiée au Journal Officiel en février 2008, prévoit la disparition du secteur réservé et l'ouverture complète du marché à la concurrence à l'horizon 2011 (2013 pour les nouveaux Etats Membres, le Luxembourg et la Grèce).

Les directives européennes modifient profondément un secteur qui longtemps s'est organisé sous forme de monopoles nationaux. Dans ce numéro de Regards économiques, nous nous intéressons à la question du financement du service universel postal (dénommé ci-après SU) dans un environnement concurrentiel. Dans un premier temps, nous analyserons les différentes formes que peut prendre la concurrence dans le secteur postal : qui sont les opérateurs alternatifs ? Quels sont les services offerts? Quels sont les produits concernés ? Dans un deuxième temps, nous reviendrons sur le contenu des obligations de SU et sur la charge financière qui en résulte. Nous analyserons ensuite comment le financement du SU est organisé aujourd'hui (en monopole) et comment la concurrence va modifier son financement. Nous concluons l'article avec quelques considérations dans la perspective d'une libéralisation complète du marché.

\section{Quelques éléments sur la demande de courrier ${ }^{1}$}

En Belgique, durant l'année 2004, La Poste a distribué plus de 2,7 milliards d'envois, soit en moyenne 268 pièces par personne et par an. Dans ce total, ne sont

\footnotetext{
${ }^{1}$ Les données de cette section proviennent des rapports Ecorys (2005) et PwC (2006).
} 


\author{
... La concurrence dans le secteur \\ postal
}

repris que les envois adressés faisant partie du périmètre du service universel ${ }^{2}$, à l'exclusion donc des courriers non adressés (type toutes boites) qui représentent 5,8 milliards de pièces par an.

Au-delà du volume, l'analyse des flux de courrier révèle deux éléments intéressants. Premièrement, les entreprises sont les plus grands émetteurs de courrier : elles expédient $85 \%$ du volume total (tableau 1). Deuxièmement, si tout le monde (ou presque) est client de La Poste, la demande (les émetteurs de courrier) est fortement concentrée chez quelques centaines de clients. Les 350 plus gros clients de La Poste représentent en effet $35 \%$ des envois (près d'un milliard de pièces), tandis que les 2500 plus gros clients représentent $70 \%$ des envois.

Tableau 1 : flux de courrier année 2003

$\begin{array}{ll}\text { Entreprise à entreprise } & 52,1 \% \\ \text { Entreprise à particulier } & 33,2 \% \\ \text { Particulier à entreprise } & 9,5 \% \\ \text { Particulier à particulier } & 5,2 \%\end{array}$

Finalement, on distingue généralement deux catégories de courrier : le courrier égrené, préparé à la pièce, et le courrier industriel, préparé en nombre. Dans cette dernière catégorie, on retrouve les envois de type facture ou marketing direct. La collecte et le tri du courrier égrené représentent des coûts relativement importants par rapport au courrier industriel. Ce dernier est, en effet, préparé électroniquement et pré-trié par l'expéditeur. Le courrier industriel constitue la plus grande partie de la demande. Par ailleurs, dans bon nombre de cas, ce courrier n'est pas urgent.

\section{Les nouveaux opérateurs} postaux

Dans un marché ouvert à la concurrence, les nouveaux opérateurs postaux vont prioritairement cibler le courrier industriel émis en nombre par les entreprises. Cette stratégie permet de collecter des volumes relativement importants auprès d'un nombre restreint de clients. Ce faisant, les nouveaux opérateurs limitent les coûts de traitement du courrier.

Dans le périmètre du service universel, trois types d'opérateurs sont susceptibles de faire concurrence à l'opérateur historique : les consolidateurs de courrier, les nouveaux entrants "End-to-End" (E2E) et les réseaux de distribution de courrier non adressé. ${ }^{3}$

Les consolidateurs de courrier sont actifs dans les segments amonts du marché (la collecte, le transport et le tri) et ne disposent pas d'un réseau de distribution. Leur objectif est de massifier le courrier pour bénéficier de tarifs préférentiels. Les envois traités par les consolidateurs sont finalement remis à l'opérateur historique qui se charge de la distribution finale. Certains consolidateurs comme UKMail en Angleterre parviennent à atteindre des volumes conséquents (5\% du marché).

L'ouverture du marché à la concurrence a permis l'arrivée de nouveaux opérateurs E2E sur le marché. Ceux-ci offrent un service complet à leurs clients : ils assurent eux-mêmes toutes les opérations postales, de la collecte à la distribution. Leur cible est le courrier industriel pré-trié et non urgent. Ces nouveaux opérateurs adoptent un modèle original : le courrier n'est pas distribué quotidiennement mais deux à trois fois par semaine. De plus, ces entreprises E2E ne sont pas tenues de desservir l'entièreté du territoire. Par exemple, CityMail ne couvre que

\footnotetext{
${ }^{2}$ Le courrier de moins de $2 \mathrm{~kg}$ et les colis de moins de $10 \mathrm{~kg}$.

${ }^{3}$ On observe également l'arrivée d'opérateurs alternatifs dans certaines niches du marché, les courriers échangés entre médecins par exemple.
} 


\section{... La concurrence dans le secteur postal}

\section{Le service universel postal}

$40 \%$ de la population au Danemark et $45 \%$ de la population en Suède. Aux PaysBas, les deux nouveaux opérateurs E2E distribuent le courrier dans tout le pays mais leurs réseaux se sont mis en place progressivement. Ces nouveaux opérateurs parviennent à l'équilibre économique avec des parts de marché relativement faibles $(8-10 \%)$.

Avec la disparition des secteurs réservés, les distributeurs de courrier non adressé peuvent étendre leurs activités au courrier adressé. Ces entreprises disposent déjà d'un réseau de distribution et d'importants volumes d'envois. L'extension des activités au courrier adressé permettra à ces entreprises de bénéficier des économies d'échelle et de gammes, et de fournir ainsi un service à un coût avantageux. ${ }^{4}$ Les distributeurs de courrier peuvent, comme les opérateurs E2E, limiter leur couverture géographique. C'est le cas par exemple d'Adrexo en France qui ne couvre que $40 \%$ du pays. En Belgique, par contre, le principal distributeur de toutes boites (Belgique Diffusion, filiale de TNT) dessert toutes les adresses et a déjà annoncé son intention d'étendre ses services au courrier adressé. ${ }^{5}$

L'arrivée d'un ou plusieurs concurrents sur le marché modifie le financement du service universel postal. En se concentrant sur les segments les plus rentables du marché, la charge du SU laissée à l'opérateur historique ${ }^{6}$ augmente et le financement du SU pourrait ne plus être assuré.

\section{La définition des obligations de service universel}

Les obligations de service universel se définissent selon trois axes : (1) le périmètre du SU, (2) les obligations en termes de qualité du service et (3) les contraintes en termes de prix. L'importance de ces différentes obligations varie considérablement de pays à pays.' Ceci n'est évidemment pas sans influence sur le coût du SU.

(1) Périmètre du service universel. Les opérateurs postaux sont des entreprises multi-produits. Les contraintes de service universel ne s'appliquent qu'à un nombre restreint de ces produits. Ceux-ci se définissent par poids, par dimension et par type de produit. La première directive postale prévoit que le courrier de moins de $2 \mathrm{~kg}$ et les colis de moins de $10 \mathrm{~kg}$ fassent partie du SU mais les États ont également la possibilité d'étendre le périmètre du SU.

(2) Qualité du service. Elle se mesure par différents paramètres : la fréquence des collectes, la fréquence de la distribution, l'accessibilité des points de collecte et de contact et les délais d'acheminement. La directive de 1997 définit des obligations minimales, notamment une collecte et une distribution journalière sur l'ensemble du territoire. Les Etats Membres doivent préciser le contenu des obligations en termes de qualité du service.

(3) Contraintes tarifaires. On retrouve deux types de contraintes sur les prix : l'une sur le niveau des prix, les produits qui composent le service universel devant être fournis à un prix abordable, l'autre sur la structure des prix, à savoir l'imposition d'un tarif uniforme sur l'ensemble du territoire pour certains produits.

\footnotetext{
$\overline{{ }^{4} \text { On parle d'économies d'échelle lorsque le coût unitaire diminue avec le volume de courrier. On parle d'économies de gamme (ou }}$ d'envergure) lorsque le coût unitaire diminue avec le nombre de produits distribués. Un réseau de distribution postal exhibe à la fois des économies d'échelle et de gamme.

${ }^{5}$ Source : PwC (2006).

${ }^{6}$ Ou plus précisément à l'opérateur qui se voit imposer les obligations de SU. Il n'est pas exclu qu'à terme, une autre entreprise que l'opérateur historique soit désignée comme prestataire du service universel.

${ }^{7}$ Voir à ce sujet Ambrosini, Boldron et Roy (2006).
} 


\section{... Le service universel postal}

\section{Mesurer \\ la charge financière du service universel}

La première directive postale définit un socle minimal d'obligations. Le contenu précis des obligations de SU est en grande partie laissé à l'appréciation des Etats Membres. Ceux-ci décident effectivement des critères de qualité du service et des contraintes tarifaires qu'ils imposent au prestataire de SU. De ce fait, l'importance des contraintes de service universel varie de pays à pays.

L'imposition d'obligations de SU entraîne à la fois des coûts et des bénéfices. Il y a donc lieu de déterminer le coût net du service universel (la différence entre les bénéfices et les coûts) et, si celui-ci représente une charge financière inéquitable (un coût net négatif), de compenser le prestataire pour les services qu'il fournit. Dans le contexte postal, l'évaluation de la charge du service universel est particulièrement complexe en raison de la nature multi-produits du secteur et des économies de gamme qui en résultent. ${ }^{8}$

Actuellement, le régulateur belge (l'IBPT) utilise la méthode Fully Distributed Cost pour évaluer la charge du service universel. ${ }^{9}$ L'ensemble des coûts est entièrement alloué aux différents produits. On calcule ensuite pour chaque produit composant le service universel son coût, le revenu qu'il génère et le profit ou la perte correspondante. En prenant la somme des pertes et des profits pour l'ensemble des produits du SU, on obtient le coût net du service universel. Si le résultat final est positif, les obligations de SU sont autofinancées. S'il est négatif, l'imposition des obligations de SU représente une charge inéquitable qu'il y a lieu de compenser. Dans sa troisième directive, la Commission européenne propose une méthode de calcul basée sur la construction d'un scénario contrefactuel : le coût net correspond à la différence entre le coût net supporté par le prestataire de SU lorsqu'il est soumis aux obligations de service universel et celui qui est supporté par le même prestataire lorsqu'il n'est pas soumis à ces obligations. ${ }^{10}$

Finalement, dans un marché libéralisé où plusieurs opérateurs sont en concurrence, il est possible d'attribuer par une procédure de type appel d'offres les obligations de service universel. La mise en concurrence d'opérateurs permet de sélectionner l'entreprise qui est à même de fournir le service au moindre coût. De plus, le coût net du SU est lui aussi déterminé par le marché.

Plusieurs facteurs déterminent la charge

du $S U$

La définition du SU et de la méthode de calcul de son coût net ne sont que deux éléments intervenants dans la détermination de la charge du SU. Le volume de courrier, les caractéristiques géographiques du pays et l'efficacité du prestataire ont également leur importance. La charge du SU dépend de :

1. De la définition du SU. Les Etats Membres ont une certaine latitude pour définir le SU. Plus ils adopteront de contraintes, plus la charge induite sera importante.

2. De la méthode choisie pour l'évaluer. En fonction de la méthode de calcul choisie, le coût net du SU peut varier. Pour cette raison, la Commission recommande une méthode de calcul aux Etats Membres.

3. Du volume de courrier. En raison des économies d'échelle, les coûts unitaires dépendent négativement du volume de courrier distribué. L'évolution de la demande ${ }^{11}$ et l'importance de la concurrence sur le marché affecteront le volume

\footnotetext{
${ }^{8}$ En présence d'économies de gamme, la distribution de produits en dehors du périmètre du SU réduit le coût de distribution des produits du SU.

${ }^{9}$ Une explication détaillée est disponible sur le site de l'IBPT : http://www.ibpt.be/.

${ }^{10}$ Voir à ce sujet Cremer, Grimaud et Laffont (2000).

${ }^{11}$ L'utilisation accrue de nouveaux modes de communication rend l'évolution de la demande de courrier incertaine.
} 


\section{... Le service universel postal}

\section{Concurrence et service universel}

de courrier distribué par le prestataire de SU et par-là la charge qui en résulte. La charge du SU n'est donc pas figée et évolue avec le marché.

4. Des caractéristiques du pays (géographie, densité de population, ...). Le coût de distribution dépend entre autres de la densité de population. Il est évidemment moindre dans les centres urbains que dans les zones rurales. Les caractéristiques géographiques du pays ont un impact important sur les coûts. Boldron, Joram, Martin et Roy (2006) estiment qu'en France, les coûts unitaires d'un envoi varient d'un facteur 1 à 5,3 en fonction de la localisation du destinataire. ${ }^{12}$

5. De l'efficacité de l'entreprise qui en a la charge. La perspective d'une libéralisation complète du marché a incité les opérateurs postaux européens (au moins certains d'entre eux) à entreprendre d'importantes restructurations afin de diminuer les coûts et d'améliorer la productivité. Citons, par exemple, l'automatisation du tri, la réorganisation du réseau de distribution et des points de vente. L'efficacité de l'opérateur historique affecte directement la charge du SU en diminuant le coût de production et indirectement en modifiant éventuellement le niveau de concurrence sur le marché.

Jusqu'à la libéralisation complète du marché en 2011 , le coût net du SU est à charge de l'opérateur historique qui le finance par les profits qu'il réalise, en ce compris les profits qu'il retire dans les segments réservés du marché dans lesquels il est protégé de la concurrence. En d'autres mots, en monopole, le coût net du SU est positif. Depuis le 1er janvier 2006, le secteur réservé ne comprend plus que le courrier de moins de 50 grammes n'excédant pas 2,5 fois le tarif minimum.

Le financement en monopole requiert des subsides croisés : les services rentables financent les services non rentables. Avec la disparition programmée des secteurs réservés disparaît une partie du financement du service universel. Les nouveaux opérateurs postaux ne sont pas tenus aux mêmes obligations et peuvent se concentrer sur les segments rentables du marché (courrier industriel, distribution dans les zones denses, distribution à date fixe) laissant les segments les moins rentables au prestataire de service universel. Ils capturent ainsi une partie des profits qui auparavant finançaient le SU. En revanche, les pertes réalisées dans les segments les moins rentables restent entièrement à charge de l'opérateur historique. C'est ce qu'on appelle l'écrémage du marché (voir encadré).

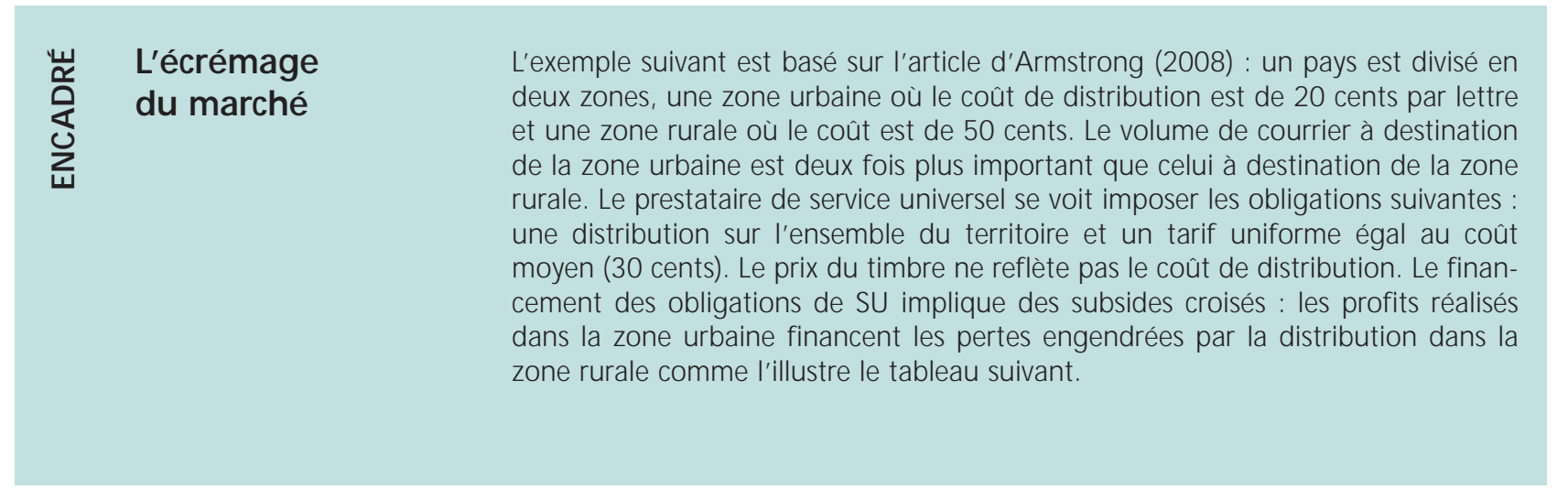

\footnotetext{
${ }^{12}$ d'Alcantara et Gautier (2008) étudient spécifiquement l'influence de la configuration d'un pays sur le niveau de concurrence et
} sur le financement du service universel. 


\begin{tabular}{lcc}
\hline & Zone urbaine & Zone rurale \\
\hline Nombre de lettres & $2 * 10^{9}$ & $1 * 10^{9}$ \\
\hline Coût de distribution par lettre & $0,20 €$ & $0,50 €$ \\
\hline Prix du timbre & $0,30 €$ & $0,30 €$ \\
\hline Profit & 200 millions $€$ & -200 millions $€$
\end{tabular}

L'ouverture du marché à la concurrence menace le financement du SU. En effet, une entreprise concurrente, qui n'est pas tenue de respecter les obligations de SU, peut opérer uniquement dans la zone urbaine. Elle y réalisera un profit positif si son coût de distribution est inférieur au prix du timbre. ${ }^{13}$ En s'installant sur le marché urbain, l'entreprise concurrente capture une partie des profits urbains qui subsidiaient la distribution en zone rurale et laisse intactes les pertes réalisées dans la zone rurale. C'est le phénomène d'écrémage du marché : les concurrents ne servent que les segments les plus attractifs (la crème), laissant les segments les moins attractifs ou déficitaires à l'opérateur en charge du SU qui ne peut pas les abandonner. Avec la concurrence, l'opérateur en place n'est plus à même de remplir ses obligations de SU à savoir une distribution sur l'ensemble du territoire à un tarif uniforme.

II existe pourtant des solutions pour éviter l'écrémage du marché. La plus évidente est l'abandon du tarif uniforme. Sans ce tarif uniforme, une lettre à destination de la zone urbaine coûte 20 cents, une lettre à destination de la zone rurale coûte 50 cents et il n'y a plus de subsides croisés. Une autre solution consiste à établir un fonds de compensation : chaque opérateur qui distribue une lettre dans la zone urbaine contribue à hauteur de 10 cents au fonds. Le profit du fonds est donc de 200 millions d'euros. Chaque opérateur qui distribue une lettre dans la zone rurale reçoit un payement du fonds égal à 20 cents. Les pertes de 200 millions qu'implique la distribution en zone rurale sont donc épongées par le fonds de compensation. L'idée du fonds de compensation est de découpler les subsides pour les segments non-rentables ou moins rentables des profits de l'opérateur historique. Avec un tel fonds de compensation, même si l'opérateur historique perd tous ses clients sur le marché urbain, la distribution en zone rurale au prix de 30 cents est toujours assurée. ${ }^{14}$

L'intensité du problème de financement dépendra à la fois de l'importance des obligations de service universel et de l'importance de la concurrence sur le marché. En l'absence de service universel, il n'y a évidemment pas besoin de se poser la question de son financement. En l'absence de concurrence, il n'y a pas besoin d'un mécanisme de financement. La concurrence en tant que telle n'est d'ailleurs pas problématique. Au contraire, elle devrait être source d'innovations et de gains d'efficacité au profit des consommateurs. Cependant, la conjonction du service universel et de la concurrence pourrait s'avérer problématique.

L'importance de la concurrence sur le marché dépend de l'efficacité de l'opérateur en place et de caractéristiques propres à chaque pays, en particulier des caractéristiques géographiques (urbanisation, densité de population, ...) et des caractéristiques du marché (nombre d'envois par habitant, composition de la

\footnotetext{
${ }^{13}$ Et non pas inférieur au coût de l'opérateur historique. Le tarif uniforme qui découple prix et coût donne de mauvais signaux aux entrants. Une entreprise moins efficace qui aurait par exemple un coût de distribution en zone urbaine de 25 cents peut entrer sur le marché urbain et réaliser des profits. De même, une entreprise plus efficace qui aurait un coût de distribution de 40 cents en zone rurale ne pourrait pas profitablement entrer sur le marché.

${ }^{14}$ En présence du fonds de compensation, une entreprise ne peut entrer sur le marché que si elle est plus efficace que l'opérateur en place.
} 


\author{
... Concurrence et service \\ universel
}

demande, sensibilité de la demande). Les parts de marché des nouveaux opérateurs postaux sont donc susceptibles de varier fortement d'un pays à l'autre.

Plusieurs études tentent d'estimer la couverture de marché des entrants en fonction des caractéristiques d'un pays. Par exemple, d'Alcantara et Gautier (2008) considèrent trois pays hypothétiques qui ne diffèrent que par la localisation des habitants dans les zones denses et moins denses, les autres paramètres (coût unitaire, demande) étant constants. Dans ce modèle, la part de marché de l'opérateur alternatif varie de $2 \%$ à $30 \%$ et sa couverture géographique de $3,5 \%$ à $45 \%$ des adresses. Avec, évidemment, des conséquences radicalement différentes sur la charge du SU et son financement. L'étude PwC (2006) intègre d'autres dimensions dans l'analyse, comme le volume de courrier, et estime pour chaque Etat Membre les parts de marché des nouveaux opérateurs postaux. Dans cette étude, la Belgique se trouve dans le groupe de pays où la part de marché estimée qu'atteignent les nouveaux opérateurs après l'ouverture du marché est la plus importante. Avec comme corollaire, l'existence d'un besoin de financement pour le service universel modéré ou limité en fonction de la définition du service universel retenue. $^{15}$

Si les obligations de SU représentent une charge inéquitable après l'ouverture du marché à la concurrence, l'opérateur est en droit de recevoir une compensation pour les prestations qu'il effectue. La troisième directive postale prévoit plusieurs possibilités pour compenser le prestataire de service universel. Le choix d'un mécanisme de financement, comme la définition du SU, est laissé aux Etats membres.

La première possibilité est de faire appel aux fonds publics. Dans ce cas, la charge du SU est financée par le gouvernement qui impose les obligations de SU à l'opérateur. L'Etat paye pour les services déficitaires qu'il contraint de prester. La seconde possibilité consiste à répartir la charge du service universel sur l'ensemble des entreprises actives sur le marché. Cela peut se faire en créant un fonds de compensation, alimenté par les opérateurs postaux proportionnellement à leur chiffre d'affaires ou au volume de courrier distribué. Les fonds collectés servent à financer la charge du SU. C'est l'option qui a été retenue en France sans que le fonds ne soit encore activé. Au lieu du fond de compensation, on peut mettre en place un mécanisme de type "pay-or-play" où les opérateurs postaux ont le choix entre se conformer aux exigences du service universel ou participer à son financement.

L'imposition des obligations de SU peut conduire à une situation où le prestataire se trouve dans une situation inéquitable sur le marché face à ses concurrents. La possibilité d'une compensation pour la charge du SU vise à corriger ce désavantage concurrentiel. Une alternative consiste à imposer des obligations de SU à tous les opérateurs présents sur le marché, par exemple l'obligation de couvrir tout le territoire. La directive postale prévoit la mise en place d'un système de licence pour les opérateurs postaux et la possibilité de subordonner l'autorisation d'opérer sur le marché à des obligations de SU.

La Suède (en 1993), le Royaume-Uni (en 2006) et l'Allemagne (en 2008) ${ }^{16}$ ont devancé l'application de la troisième directive postale et ont entièrement libéralisé leur marché. En Angleterre et en Suède, la concurrence des nouveaux opérateurs est intense, même si l'opérateur historique maintient des parts de marché conséquentes. Dans ces deux pays, le service universel continue d'être assuré sans

\footnotetext{
${ }^{15}$ Le maintien ou non de l'obligation de tarif uniforme pour le courrier industriel.

${ }^{16}$ L'ouverture totale du marché en Allemagne s'est accompagnée d'une loi qui fixe le salaire horaire minimum dans le secteur postal à $9,80 €$. Ce salaire minimum est en dessous du salaire minimum offert par Deutsche Post $(11,40 €)$ mais au-dessus du salaire moyen des principaux concurrents $(8,44 €)$ (source : Dieke et Wojtek, 2008). Le niveau du salaire minimum est contesté par les deux principaux opérateurs alternatifs qui ont (provisoirement?) renoncé à mettre en place un réseau de distribution à grande échelle.
} 


\author{
... Concurrence et service \\ universel
}

\section{Perspectives 2011}

que l'opérateur historique ne reçoive de soutien financier de l'Etat ou de ses concurrents.

En Suède, il existe un opérateur concurrent E2E (CityMail) sur le segment du courrier industriel, même si celui-ci ne couvre qu'une partie du territoire. Depuis la libéralisation du secteur, on observe que sur le segment concurrentiel du marché (le courrier industriel), le prix a diminué tandis que sur le segment monopolistique (le courrier égrené), le prix a augmenté. Le financement du SU continue d'être assuré sans mécanisme de compensation mais la charge du SU s'est donc déplacée d'un segment du marché vers l'autre. Le prix diminue sur les segments de marché où les clients ont le choix entre plusieurs fournisseurs. Il augmente pour les clients captifs qui n'ont pas d'autres solutions. ${ }^{17}$

Comme nous l'avons expliqué, l'ouverture du marché à la concurrence modifie la charge du service universel et son mécanisme de financement. Les Etats Membres doivent prendre un certain nombre de mesures pour encadrer le marché et assurer la pérennité du service universel. Il faut d'abord définir ou re-définir les obligations de service universel. A ce titre, il est sans doute légitime de se demander si les obligations imposées actuellement doivent être maintenues. On pourrait, par exemple, supprimer l'obligation de tarif uniforme pour certains produits du service universel, d'autant plus que les nouveaux opérateurs ne seront pas soumis à cette obligation. En limitant les contraintes, on limite également le besoin de financement. Et, de même qu'on peut s'interroger sur la structure des prix, on peut s'interroger sur leurs niveaux. Comment définir un tarif abordable pour les produits du SU ? L'exemple suédois montre que la concurrence entraîne une modification de la structure tarifaire : le courrier industriel devient relativement moins cher; le courrier égrené devient relativement plus cher. S'il y a une régulation des prix, quels sont les prix concernés ? Faut-il réguler le prix de tous les produits dans le périmètre du SU ou se limiter au courrier égrené ? Une fois le SU clairement défini, il faut s'accorder sur le calcul de la charge qu'il représente. Finalement, un mécanisme de compensation doit être mis en place et activé si cela s'avère nécessaire. Un tel mécanisme doit à la fois préserver la concurrence sur le marché ${ }^{18}$ tout en assurant le financement du service universel.

En Belgique, le volume élevé de courrier par habitant et l'importance du courrier industriel rendent le marché particulièrement attractif pour les opérateurs concurrents. On peut donc s'attendre à une concurrence intense sur certains segments du marché, au plus grand bénéfice des consommateurs. Par ailleurs, les contraintes de service universel sont relativement importantes. Comme nous l'avons expliqué dans cet article, la conjonction de ces deux facteurs peut s'avérer problématique. Sans doute plus que dans d'autres pays, le choix et la mise en place d'un mécanisme qui assurera le financement du service universel vont revêtir une importance capitale. Plusieurs options sont envisagées et feront l'objet de débats dans les mois et les années à venir. ${ }^{19}$ La re-définition des obligations de SU et

\footnotetext{
${ }^{17}$ Le prestataire de SU se trouve face au problème suivant : des coûts communs (par exemple, les coûts de distribution) doivent êtres répartis entre plusieurs produits pour lesquels la demande diffère, la répartition des coûts étant reflétée par après dans le prix du produit. Les principes de tarification optimale recommandent que la part des coûts supportée par chaque produit soit inversement proportionnelle à l'élasticité (la sensibilité) de la demande par rapport au prix. Les produits pour lesquels la demande est peu sensible au prix supportent une part plus importante des coûts (et sont donc vendus à un prix relativement plus élevé) que les produits dont la demande est fort sensible au prix. L'évolution des tarifs suédois reflète (en partie) le principe de tarification optimale. ${ }^{18}$ Le système de licence payante mis en place en Finlande a pour le moment dissuadé tous les concurrents d'entrer sur le marché. ${ }^{19}$ L'autre question qui fera certainement débat dans notre pays est celle de l'intégration ou non de tous les travailleurs du secteur postal au sein d'une même convention collective sectorielle.
} 
notamment les contraintes en termes de prix et de tarification font partie intégrante de ce débat sur l'avenir du SU. Idéalement, le mécanisme mis en place doit assurer le financement du SU tout en ayant un impact limité sur le niveau de concurrence. Si ce n'est pas possible, il faudra faire un arbitrage entre ces deux dimensions, sous l'œil attentif de la Commission européenne qui sera particulièrement attentive à ce que les options choisies ne limitent pas (trop) la concurrence sur le marché.

Axel Gautier est professeur

Axel Gautier

d'économie à HEC-ULg et membre

$d u$ CORE (UCL).

\section{Références}

Ambrosini, X., F. Boldron et B. Roy (2006) "Universal Service Obligations in the Postal Sector: Economics Learnings from Cross-Country Comparisons" in M. A. Crew a P. R. Kleindorfer (eds), Progress toward Liberalization of the Postal and Delivery Sector, Springer.

Armstrong, M. (2008) “Access Pricing, Bypass and Universal Service in Post”, The Review of Network Economics 7(2), pp 172 - 187.

Boldron, F., D. Joram, L. Martin et B. Roy (2006) "From Size of the Box to the Costs of Universal Service Obligation: A Cross-Country Comparison", in M. A. Crew a P. R. Kleindorfer (eds), Liberalization of the Postal and Delivery Sector, Edward Elgar.

Cremer, H., A. Grimaud et J.J. Laffont (2000) "The cost of universal service in the postal sector" in M. A. Crew, and P. K. Kleindorfer (eds.), Current Directions in Postal Reform, Kluwer Academic.

d'Alcantara, G. et A. Gautier (2008) "National Postal Strategies after Full Postal Market Opening", in M. A. Crew, and P. K. Kleindorfer (eds.), Competition and Regulation in the Postal and Delivery Sector, Edward Elgar.

Dieke, A. K. et R. Wojtek (2008) "Competition, wages and politics in the delivery sector: The case of postal minimum wages in Germany", article présenté à la 16th Conference on Postal and Delivery Economics, Albufeira, Portugal.

Ecorys (2005) "Developpment of Competition in the European Postal Sector", rapport final.

http://ec.europa.eu/internal_market/post/doc/studies/2005-ecorys-final_en.pdf

PricewaterhouseCoopers (2006) "The Impact on the Universal Service of the Full Market Accomplishment of the Postal Internal Market in 2009", rapport final et annexes.

http://ec.europa.eu/internal_market/post/doc/studies/2006-impact-report_en.pdf http://ec.europa.eu/internal_market/post/doc/studies/2006-impactannexes_en.pdf 
Directeur de la publication :

Vincent Bodart

Rédactrice en chef :

Muriel Dejemeppe

Comité de rédaction : Paul Belleflamme,

Vincent Bodart, Thierry Bréchet,

Muriel Dejemeppe, Frédéric Docquier,

Jean Hindriks, François Maniquet,

Marthe Nyssens

Secrétariat \& logistique : Anne Davister

Graphiste : Dominos

\section{Regards Économiques}

\section{IRES-UCL}

Place Montesquieu, 3

B1348 Louvain-la-Neuve

http://www.uclouvain.be/regardseconomiques

regard-ires@uclouvain.be.

tél. 010/47 3426 